\title{
Part-whole information is useful in visual search for size $\times$ size but not orientation $\times$ orientation conjunctions
}

\author{
ALEXANDER B. BILSKY \\ University of Massachusetts, Amherst, Massachusetts \\ and \\ JEREMY M. WOLFE \\ Center for Ophthalmic Research, Brigham and Women's Hospital and Harvard Medical School \\ Boston, Massachusetts
}

\begin{abstract}
In visual search tasks, subjects look for a target among a variable number of distractor items. If the target is defined by a conjunction of two different features (e.g., color $x$ orientation), efficient search is possible when parallel processing of information about color and about orientation is used to guide the deployment of attention to the target. Another type of conjunction search has targets defined by two instances of one type of feature (e.g., a conjunction of two colors). In this case, search is inefficient when the target is an item defined by parts of two different colors but much more efficient if the target can be described as a whole item of one color with a part of another color (Wolfe, Friedman-Hill, \& Bilsky, 1994). In this paper, we show that the same distinction holds for size. "Partwhole" $^{\prime}$ size $\times$ size conjunction searches are efficient; "part-part" searches are not (Experiments $1-3$ ). In contrast, all orientation $\times$ orientation searches are inefficient (Experiments 4-6). This difference between preattentive processing of color and size, on the one hand, and orientation, on the other, may reflect structural relationships between features in real-world objects.
\end{abstract}

Finding one item among many is a task that is faced frequently and solved routinely by the visual system. In laboratory versions of these visual-search tasks, subjects are presented with a variable number of items and must decide whether or not a designated target item is present among the distractor items. Search efficiency is constrained by the way in which the target differs from the distractors. For instance, if some feature of the target is unique, say, color, size, or orientation, and if the distractor items are homogeneous, search is usually very efficient. Indeed, these "feature" searches often appear to occur "in parallel," with all items processed at once (Treisman \& Gormican, 1988). In more natural settings, such pure feature-search tasks are comparatively rare. Natural targets are unlikely to be the orily vertical item or the only curved item in the field. It is more likely that a target will be defined by the conjunction of two or more basic features (e.g., the small, red, shiny object in a field where other objects may be small or red or shiny but will not show all three attributes). In such searches, the results of the parallel processing of each basic

This research is supported by NIH-NEI Grant RO1-EY05087 to J.M.W. and by AFOSR Grant F49620-93-1-0407 to J.M.W. We thank Patricia O'Neill, Greg Gancarz, Ruth Kimchi, and Sara Bennett for comments on various drafts of this paper and Stacia Friedman-Hill for help in collecting, analyzing, and thinking about the data. Correspondence should be addressed to J. M. Wolfe, Center for Ophthalmic Research, Brigham and Women's Hospital, 221 Longwood Ave, Boston, MA 02115 (e-mail: wolfe@search.bwh.harvard.edu). feature can be combined to guide attention to conjunctive targets (Wolfe, 1994; Wolfe, Cave, \& Franzel, 1989). Studies of a variety of conjunction searches have revealed that the ability to combine feature information has some limitations (Wolfe et al., 1990). For example, while attention can be guided to conjunctions of color and orientation, search is very inefficient when the target is defined by the conjunction of two colors or two orientations. Thus, search is very inefficient when the target is a red and green item among distractor items that are either red and blue or blue and green. Recently, Wolfe, Friedman-Hill, and Bilsky (1994) showed that this limitation does not hold when the two colored regions are in a hierarchical relationship to each other. While it is difficult to find a target defined by its adjacent red and green regions, it is comparatively easy to find a red whole item with a green part. Part-whole color $\times$ color searches are about as efficient as searches for a target defined by a conjunction of two different dimensions (e.g., color $\times$ orientation). Apparently, configural information about the structure of an item can be extracted from the display in parallel and used to guide the deployment of attention. ${ }^{1}$ Wolfe et al. (1994) reported only on color $\times$ color conjunctions. The experiments reported in this paper examined size $\times$ size and orientation $\times$ orientation conjunctions. The experiments support three conclusions: (1) Search for nonhierarchical size $\times$ size conjunction targets is very inefficient. Size is similar, in this regard, to color and orientation (Wolfe et al., 1990). (2) Search for hierarchical size $\times$ size conjunction targets 
is reasonably efficient. Structure of items influences parallel processing of size as it influences parallel processing of color (Wolfe et al., 1994). (3) Orientation $\times$ orientation searches, however, remain very inefficient in all cases. Structural information is not useful in the parallel processing of orientation.

In typical search experiments, subjects are asked to respond as rapidly and as accurately as possible to the presence or absence of a target in a display containing a variable number of distractor items. The usual dependent measure is the slope of the function relating reaction time (RT) to set size, that is, the number of items presented. This slope is a measure of the efficiency of the search, with shallower slopes corresponding to more efficient search. In some visual search tasks, like the simple feature searches described above, RT does not increase as the number of distractors increases. If the time it takes to process a large number of items is no longer than the time it takes to process a small number of items, then it is generally assumed that all items are being processed essentially simultaneously-in parallel.

If each item in the display must be attended to individually, RT will increase as the number of items in the display increases. If such a search ends either when the target is found or, on target-absent trials, when all items have been examined, then the search is said to be serial and self-terminating. The hallmarks of such searches are a linear increase in RT with set size and a 2:1 ratio between target-absent and target-present slopes (Kwak, Dagenbach, \& Egeth, 1991; Treisman \& Gelade, 1980; but see Townsend, $1990^{2}$ ). Using stimuli that are easily discriminated once they are attended to (e.g., Ts and Ls), slopes of serial, self-terminating searches tend to be $20-30 \mathrm{msec} /$ item for target-present trials and about twice that for target-absent trials, suggesting that each item takes about $50 \mathrm{msec}$ to process.

Guided searches for conjunctions of two or more basic features produce average target-trial slopes that range from near 0 to $10-12 \mathrm{msec} / \mathrm{item}$ (Wolfe et al., 1989). In these searches, the parallel processing of feature information restricts the serial deployment of attention to a subset of the items. Within that subset, search proceeds at the same $50-\mathrm{msec} / \mathrm{item}$ rate but, since some items do not need to be examined, the average cost per items drops (details in Wolfe, 1994).

In the inefficient color $X$ color and orientation $X$ orientation searches described in Wolfe et al. (1990), slopes were "superserial," about $40 \mathrm{msec} / \mathrm{item}$ for the targetpresent trials and nearly twice that for the blank trials. Perhaps this reflects a longer "dwell time" at each item for these stimuli. In contrast, the more efficient, partwhole or hierarchical color $\times$ color searches produced average slopes in the guided range, around $10 \mathrm{msec} /$ item (Wolfe et al., 1994). Such slopes are comparable to those reported by Wolfe et al. (1989) for searches for conjunctions of color and orientation.

The first three experiments in this paper examined whether or not nonhierarchical, part-part size $\times$ size conjunction searches, like nonhierarchical color $\times$ color and orientation $x$ orientation conjunction searches, were inefficient. In addition, these three experiments examined whether or not information about part-whole relationships could be used to guide attention in size $X$ size conjunction searches, as has been found for color $X$ color conjunction searches. The final three experiments examined whether or not part-whole relationships would aid search in orientation $\times$ orientation conjunction searches.

\section{EXPERIMENT 1 Size $\times$ Size Conjunctions}

Wolfe et al. (1990) examined within-feature conjunction searches only for color and orientation. In Experiment 1 , we examined size $\times$ size conjunction searches using "snowmen" stimuli composed of two circles, one directly above the other (see Figure 1). The size of the head (the top circle), the size of the body (the bottom circle), and the size of the checks on the body were variable. In the part-part condition, the relevant stimulus regions were two roughly coequal parts of the item. Subjects searched for the snowman with the small head and the big body among distractors with a small head and a smail body or a big head and a big body. The partpart condition was designed to test the hypothesis that

\section{Part-Part Condition}

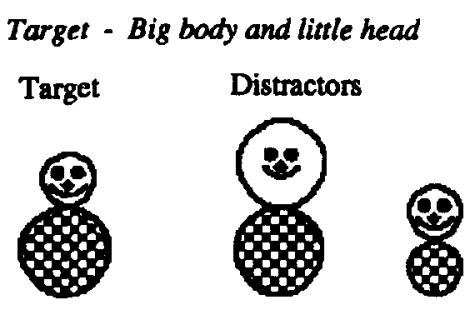

\section{Part-Whole Condition}

Target - Big body and little checks

Target Distractors

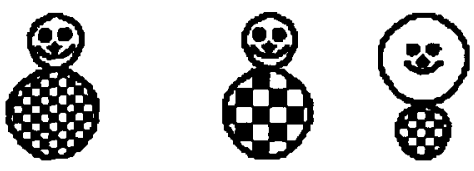

\section{Feature Search Condition}

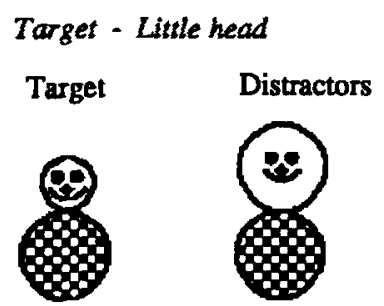

Figure 1. Stimuli for Experiment 1: Size $\times$ size conjunctions. 
nonhierarchical, part-part size $\times$ size conjunction searches would be inefficient, as was the case with partpart color $\times$ color and orientation $\times$ orientation searches. In the part-whole condition, the relevant stimulus regions did have a hierarchical relationship to one another. Among snowmen with big bodies and big checks or small bodies and small checks, subjects searched for the snowman with the big body with small checks. If size $\times$ size conjunction searches follow the same rules as color $X$ color conjunction searches, search performance in the part-whole condition should be efficient.

\section{Method}

Stimuli. Three conditions were run, as shown in Figure 1. Individual items were "snowmen" defined by three size parameters: the size of the head, the size of the body, and the size of the checks on the body. At the $62-\mathrm{cm}$ viewing distance, a large head (or body) had a $1.4^{\circ}$ diameter. A small head (or body) had a $0.8^{\circ}$ diameter. Large checks were $0.4^{\circ}$ on a side, while small checks were $0.2^{\circ}$ on a side.

Specifically, the conditions were: Part-part-Target: snowman with little head and big body. Distractors: little head/little body and big head/big body. The checks were small in all cases, so the task depended on the relationship between the two parts of the whole snowman. Part-whole-Target: big body with little checks. Distractors: big body with big checks, little body with little checks. The head was big when the body was small and was small when the body was big, so that the overall size of the target would not set it apart from either distractor. Here the task depended on the relationship between the size of the body (now the "whole") and the size of the checks (the "parts") that were on the body. Feature-Target: little head. Distractor: big head. The body was big and the checks were small in all cases.

Procedure. The stimuli were presented on a MacII computer using VSearch software (Enns, Ochs, \& Rensink, 1990). The items were presented in 4,10 , or 16 random locations in a jiggled $4 \times 4$ array of possible locations. The stimulus array subtended an area of $11.8^{\circ} \times 11.8^{\circ}$. The luminance of the background was $24.6 \mathrm{~cd} / \mathrm{m}^{2}$, while the luminance of the white regions in the items was $39.1 \mathrm{~cd} / \mathrm{m}^{2}$. The luminance of the black regions was too low to be measurable. A target item was present in the display on one half of the trials. The subjects responded with a keypress to indicate target presence or absence. Response accuracies and reaction times (RTs) were recorded by the computer. Stimuli were present until the subject responded (with a maximum duration of $5,685 \mathrm{msec}$ ). If a subject failed to respond within this maximum presentation time, RT was not recorded and that trial was counted as an error. The subjects were tested for 30 practice and 300 experimental trials in each condition. Equal numbers of trials were presented for each set size. Within the constraints already mentioned, target presence and set size were random across trials within a condition. Order of conditions was pseudorandom across subjects.

Subjects. Ten 18-38-year-old volunteer subjects were tested. All had corrected or uncorrected acuity of at least $20 / 25$ and normal color vision (Dvorine plates). All subjects gave informed consent and were paid for their participation.

\section{Results and Discussion}

Average RT $\times$ set-size functions for all three conditions are shown in Figure 2. Search in the part-part condition was very inefficient (mean slopes $=41.7 \mathrm{msec} /$ item for target trials and $105.1 \mathrm{msec} /$ item for blank trials). Performance was comparable to that found in part-part color $\times$ color and orientation $\times$ orientation conjunction searches (Wolfe et al., 1990), with slopes steeper than those seen in other serial searches. On the other hand, search in the part-whole condition was quite efficient, particularly for the target trials $(9.3 \mathrm{msec} /$ item for target trials and $39.5 \mathrm{msec} / \mathrm{item}$ for blank trials). Results for the part-whole condition are comparable to those obtained in guided searches for conjunctions of color and orientation (Wolfe et al., 1989) and in part-whole color $\times$ color conjunction searches (Wolfe et al., 1994). Partwhole slopes are significantly shallower than part-part slopes [ANOVA: target trials, $F(1,9)=74.94, p=.0001$; blank trials, $F(1,9)=310.07, p=.0001]$. The part-part task requires discrimination between the big and small

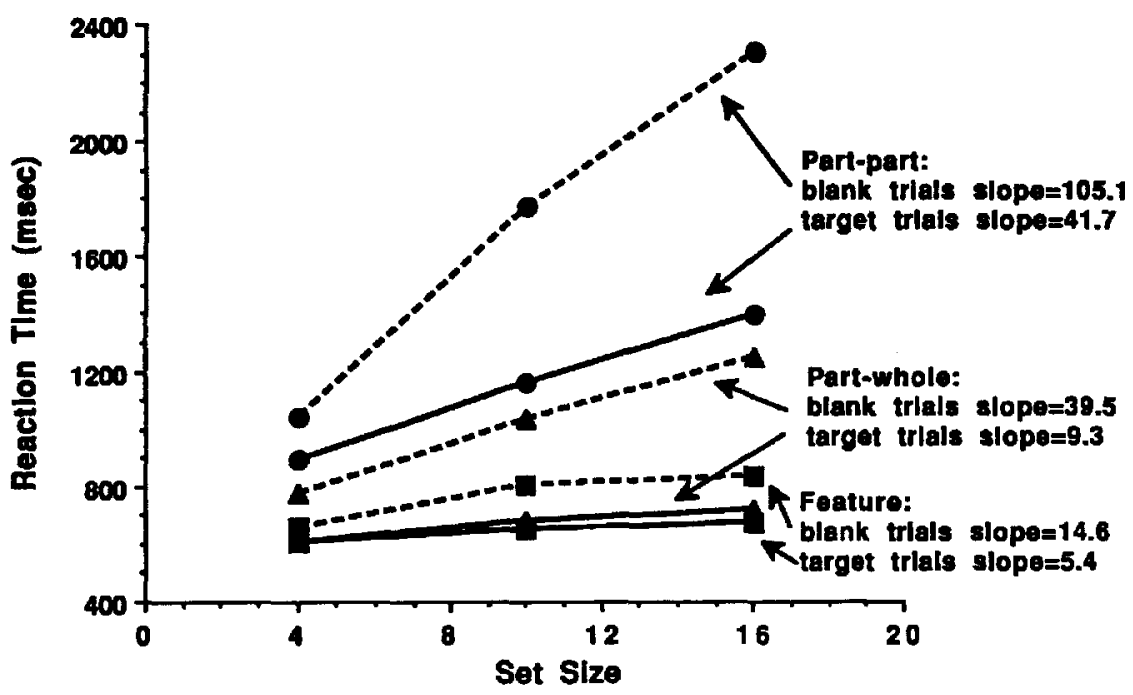

Figure 2. Results for Experiment 1: Size $\times$ size conjunctions yield steep slopes for part-part stimuli and shallower slopes for part-whole stimuli. In this and subsequent graphs, target trials are plotted with solid lines, and blank trials are plotted with dashed lines. Part-whole searches are plotted with triangles and part-part searches are plotted with circles. 
sizes of heads and bodies. The feature search tests that discrimination. The shallow slopes for that condition indicate that the difference between big and small is adequate to support efficient search. Feature searches are always more efficient than part-whole searches in these experiments.

There is substantial variability across subjects in the slope values. Individual subjects' slopes in the feature condition ranged from 0.9 to $12.2 \mathrm{msec} / \mathrm{item}$ for the target trials and from 2.5 to $44.1 \mathrm{msec} /$ item for the blank trials. In the part-whole condition, subjects' slopes ranged from 2.5 to $18.2 \mathrm{msec} /$ item on the target trials and from 12.4 to $84.3 \mathrm{msec} / \mathrm{item}$ on the blank trials. In the part-part condition, slopes ranged from 20.2 to $58.8 \mathrm{msec} / \mathrm{item}$ for the target trials and from 48.8 to $161.2 \mathrm{msec} / \mathrm{item}$ for the blank trials. Research in our laboratory has shown, however, that individual differences in target-trial slope functions for visual search tasks with similar set sizes are not reliable (O'Neill \& Wolfe, 1994), so analysis will be restricted to group average slopes, which we have found to be reliable.

Mean error rates for each condition from each experiment are shown in the Appendix. With the exception of the target trials in the part-part condition, the most difficult search condition, subjects made few errors in this experiment. On the part-part target trials, the mean error rate with set size 4 was $7.4 \%$. The error rate increased to $14.4 \%$ as the set size increased to 16 . The rising error rates reflect the increasing difficulty of the task as the set size grows, much as the rising RTs do. We assume that subjects sacrificed accuracy in an attempt to respond quickly. Had the subjects responded more slowly, the resulting slopes of the RT $\times$ set-size functions would have been even steeper in this already inefficient search.

While these results make it clear that part-whole size $X$ size conjunction searches can be efficient, they cannot prove that size part-part searches must be inefficient. This is, of course, the inevitable problem of negative evidence. It is always possible that part-part size $X$ size searches with some other set of stimuli could be efficient. The efficiency of search in the part-whole and feature conditions, however, does suggest that the stimuli in the part-part condition did contain the raw materials for efficient search. Efficient search fails when the components are conjoined in the nonhierarchical manner. In Experiment 2, we attempted to strengthen the argument that size $\times$ size part-part search tasks were necessarily inefficient.

\section{EXPERIMENT 2 A Replication}

In Experiment 2, we attempted to replicate the findings of Experiment 1 with a different set of snowman stimuli. In an attempt to make an easier part-part search, the target in the part-part condition here was a snowman with a big head and a big body. Pilot work had indicated that search for a big circle might be easier than search for a small circle. If this were true, then the search for two big circles here might be easier than the search for the big circle with the small circle in the previous experiment.

\section{Method}

The stimuli for Experiment 2 are shown in Figure 3. There are three conditions: Two size $\times$ size conjunctions-part-whole and part-part-and a feature condition. In the part-part condition, subjects searched for a snowman with a big head and big body. In the part-whole condition, subjects searched for a big body with big checks. The feature condition examined search for the big checks. The methods were otherwise identical to those in Experiment 1.

Ten new subjects were tested.

\section{Results and Discussion}

Average RT $\times$ set-size functions for Experiment 2 are shown in Figure 4. The part-part search is again very inefficient. Part-whole condition slopes are again comparable to other guided conjunction searches. Part-part slopes are significantly steeper than part-whole slopes [ANOVA on slopes: target trials, $F(1,9)=57.48, p=$ .0001 ; blank trials, $F(1,9)=338.08, p=.0001]$. Performance in the feature search is very efficient. The results here replicate the findings of Experiment 1.

Mean error rates are given in the Appendix. Again, save for the part-part target trials, the subjects made few errors in the present experiment. On part-part target trials, average errors rose from $6.6 \%$ to $15.0 \%$ as the set

\section{Part-Part Condition}

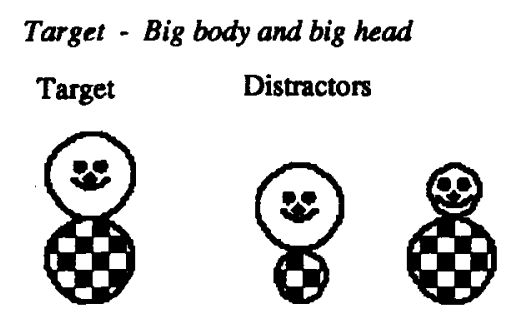

\section{Part-Whole Condition}

$$
\text { Target - Big body and big checks }
$$

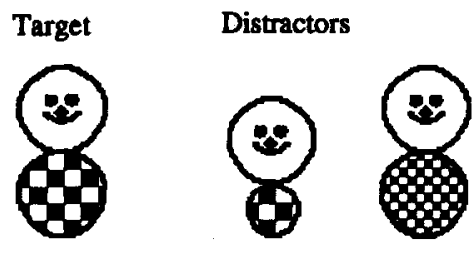

\section{Feature Search Condition}

$$
\text { Target - Big checks }
$$

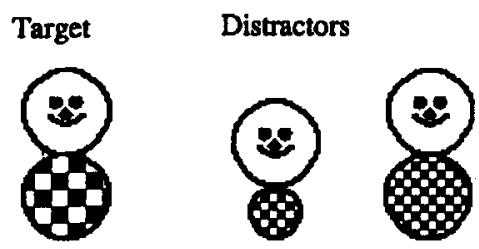

Figure 3. Stimuli for Experiment 2: Size $\times$ size conjunctions. 


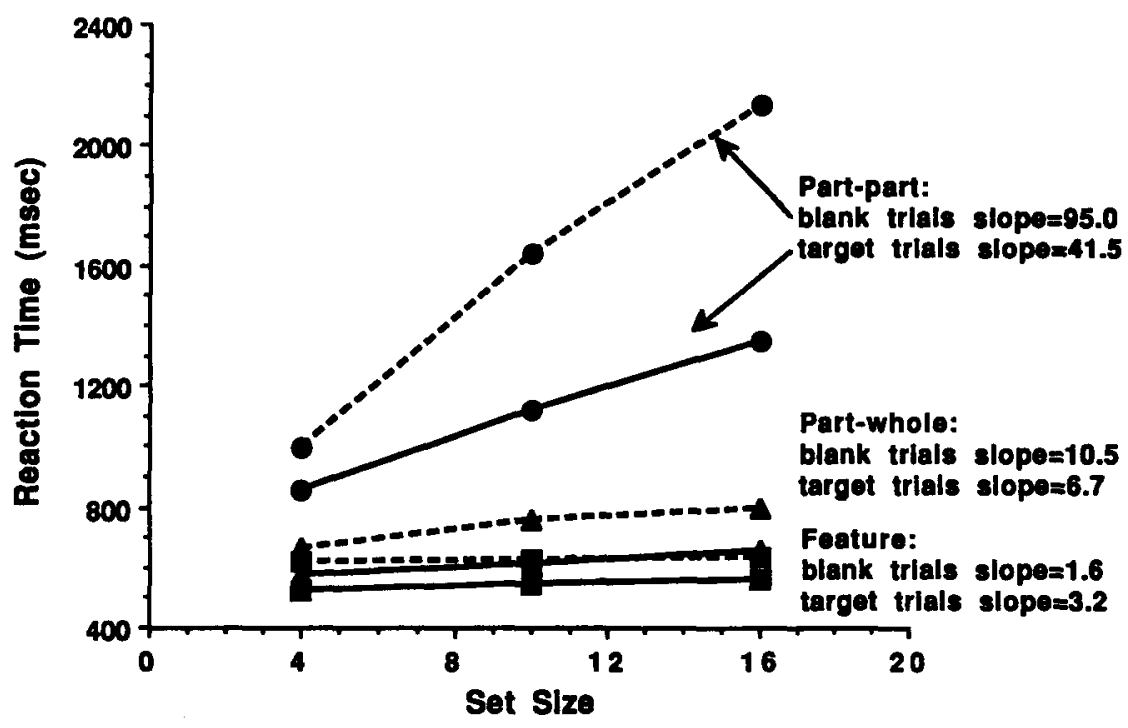

Figure 4. Results for Experiment 2: Size $\times$ size conjunctions again yield steep slopes for part-part stimuli and shallower slopes for part-whole stimuli.

size increased from 4 to 16 . The rising error rates again indicate the difficulty of the task. With a tradeoff of speed for accuracy, RT slopes would have been even higher.

\section{EXPERIMENT 3 Size $\times$ Size With Different Stimuli}

There are several possible criticisms of the snowman stimuli. First, the part-whole tasks are searches for two properties of one thing, the body, while the part-part tasks are searches for a relationship between two things, the body and the head. Duncan (1993) has demonstrated that it is easier to report two attributes of one item than one attribute of each of two items. In a sense, this is less an objection than a restatement of an important conclusion of this paper and of Wolfe et al.'s (1994). For purposes of preattentive processing, the head and the body are two different things-- two parts-even though they are clearly both parts of one object. In Experiment 3, rather than having two parts define the whole object, we use an object that happens to have two parts (see Figure 5). A second, more mundane objection to the snowman stimuli is that the part and whole are physically closer to each other than the two parts. The checks are on the body. The head is merely next to the body. Finally, a third objection might be that this is not so much a size $\times$ size conjunction in the part-whole case as it is a size $\times$ texture conjunction. Kimchi (1992), for instance, has shown that the treatment of wholes made of little, texture-like elements is different from that of wholes made of large, part-like elements. We conducted another size $\times$ size experiment using new stimuli in order to address these issues.

\section{Method}

Figure 5 illustrates the stimuli used in Experiment 3. All items were circles with two circles inside. In the part-part condition,

\section{Part-Part Condition}

Target - Big and small inner circles

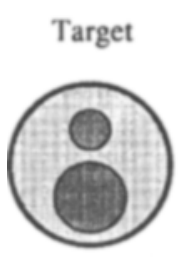

Distractors
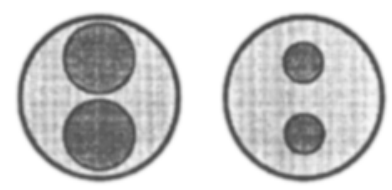

Part-Whole Condition

Target - Big outer circle with big inner circle

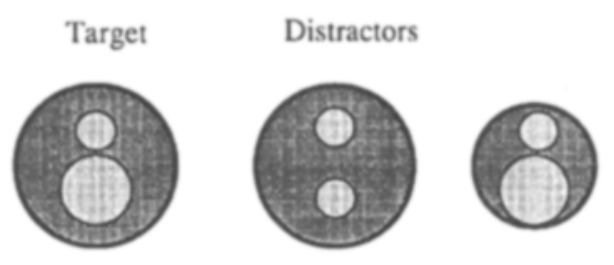

Feature Search Condition

Target - Small inner circle

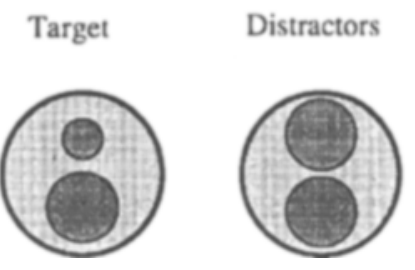

Figure 5. Experiment 3: Size $\times$ size conjunctions using stimuli where part and whole are not coextensive in the part-whole condition and where parts and wholes are all about equidistant from each other. 


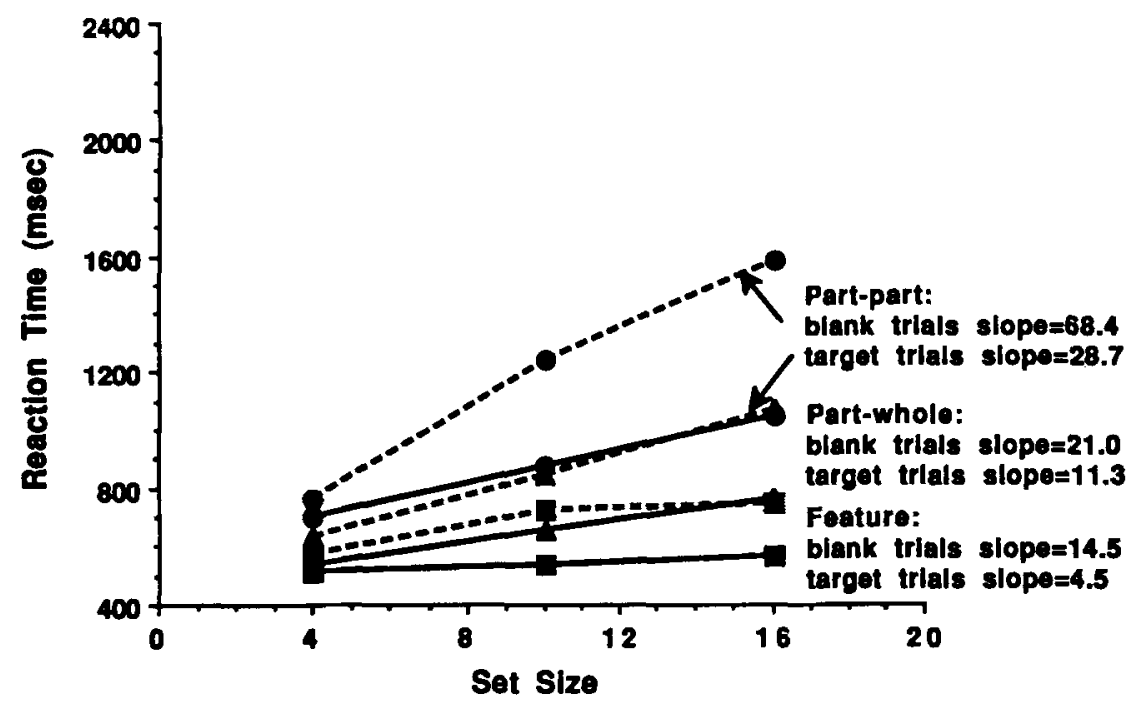

Figure 6. Results for Experiment 3, replicating the results of Experiments 1 and 2.

subjects searched for the item with big and small inner circles. In the part-whole condition, subjects searched for the big outer circle (whole) with a big inner circle (a part). In the feature condition, subjects searched for a small inner circle. In all conditions, the background on which the stimuli were presented was a light gray (luminance $18.7 \mathrm{~cd} / \mathrm{m}^{2}$ ). In the part-whole condition, the outer circle was made dark gray (luminance $9.8 \mathrm{~cd} / \mathrm{m}^{2}$ ), for greater contrast with the background, while the inner circles were again light gray. In the part-part and feature conditions, the outer circle was made the same light gray as the background and the inner circles were made dark gray. This was done to enhance the salience of the inner circles as much as possible in an effort to make the part-part condition as easy as possible. Note that (1) the part and whole are not coextensive in the part-whole condition, (2) the two parts in the part-part condition are closer to each other than the borders of the part and the whole in the part-whole condition, and (3) the parts are not textures. Viewing distance for this experiment was $57 \mathrm{~cm}$. The stimulus array subtended an area $15^{\circ} \times 15^{\circ}$. The RT cutoff was $3,000 \mathrm{msec}$. The methods were otherwise similar to those in the previous experiments.

Ten new subjects were tested.

\section{Results and Discussion}

In Figure 6, average RT $\times$ set-size functions are shown for each condition. Again, part-whole slopes were shallow and part-part slopes were steep. Part-whole search performance was significantly more efficient than partpart search performance [ANOVA on paired comparisons: target trials, $F(1,9)=51.87, p=.0001$; blank trials, $F(1,9)=373.06, p=.0001]$. Slopes in the feature search were shallow. It would seem that the advantage of part-whole over part-part tasks in the previous experiments was not due to the specific properties of the snowman stimuli.

Mean error rates are presented in the Appendix. As in the previous two experiments, error rates were low in all but the part-part target-trial cells. For those trials, error rates rose from $5.8 \%$ to $9.8 \%$ as the set size increased from 4 to 16 . Again, the somewhat high error rates reflect the difficulty of the task.
Experiments 1, 2, and 3 demonstrate first that size $x$ size conjunction searches are very inefficient when the regions of the stimuli are not in a hierarchical relationship to one another. In this way, nonhierarchical size $\times$ size conjunction searches are like nonhierarchical color $x$ color and orientation $\times$ orientation conjunction searches. Secondly, these experiments show that size $\times$ size conjunction searches can be made more efficient when hierarchical, part-whole stimuli are used. Information about hierarchical relationships between regions of items can be extracted from the stimulus in parallel and used to improve efficiency in size $\times$ size conjunction searches, as well as in color $\times$ color conjunction searches.

Experiments 4, 5, and 6 dealt with orientation $\times$ orientation conjunction searches. Wolfe et al. (1990) had already demonstrated the inefficiency of nonhierarchical orientation $X$ orientation searches. In these experiments, we used hierarchical stimuli in an effort to produce an efficient orientation $x$ orientation conjunction search. However, unlike experiments with such stimuli in the color and size domains, all of these efforts failed.

\section{EXPERIMENT 4 Rectangles and Lines}

\section{Method}

Figure 7 shows the stimuli for Experiment 4 . Stimuli were oriented rectangles with oriented bars inside. In all three conditions, the target item was a vertical rectangle with a bar tilted $45^{\circ}$ to the left inside the rectangle. In the part-whole orientation $\times$ orientation condition, the conjunction of rectangle and bar orientation defined the target. The distractors were vertical rectangles with horizontal bars inside and horizontal rectangles with tilted bars inside. In the whole-feature condition, subjects searched for the vertical rectangle whole as a feature. In the part-feature condition, subjects searched for the tilted bar part as a feature. All items were presented on a gray (luminance $11.7 \mathrm{~cd} / \mathrm{m}^{2}$ ) background. The rectangles were white, and the bars were black. The stimulus array subtended an area $15^{\circ} \times 15^{\circ}$ at the $57-\mathrm{cm}$ viewing distance. The 


\section{Part-Whole Condition}

Target - Vertical rectangle with tilted line

Target Distractors
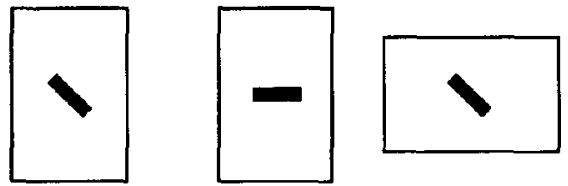

Whole Feature Condition

Target - Vertical rectangle

Target Distractors

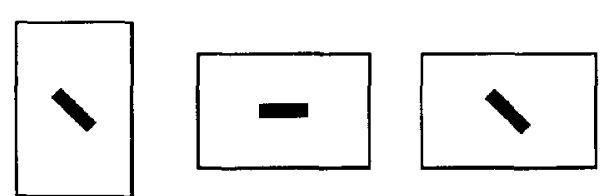

\section{Part Feature Condition}

Target - Tilted line

Target

Distractors
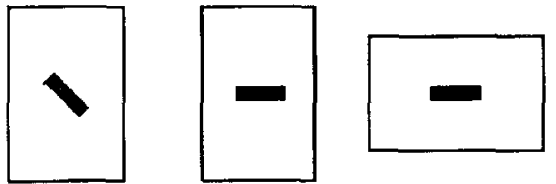

Figure 7. Stimuli for Experiment 4: Orientation $\times$ orientation conjunctions.

maximum presentation time was $3,000 \mathrm{msec}$. The methods were otherwise similar to those in the previous experiments.

Ten new subjects were tested.

\section{Results and Discussion}

Figure 8 shows average RT $\times$ set-size functions for Experiment 4. The part-whole condition slopes were far steeper than standard serial-search slopes. The 54- and $103-\mathrm{msec} / \mathrm{item}$ slopes are actually somewhat worse than the 46- and 77-msec/item slopes for the part-part orientation $\times$ orientation task shown in Figure 3 of Wolfe et al. (1990). Performance in each feature-search condition, however, was reasonably efficient. Slopes in the part-feature condition are very shallow ( $1.3 \mathrm{msec} / \mathrm{item})$, and while slopes in the whole-feature condition are somewhat steeper than typical efficient feature searches, they are still in the range of reasonably efficient search slopes $(9.2 \mathrm{msec} / \mathrm{item})$. It is interesting that the search for the vertical rectangle is as difficult as it is. A search for a vertical line among horizontal lines is usually very efficient (slope about $0 \mathrm{msec} / \mathrm{item}$ ). The presence of the bars inside the rectangles might disrupt search for rectangle orientation. Note that this sort of disruption does not seem to occur in feature searches with part-whole stimuli defined by size or color where, for instance, the color of the door does not seem to interfere with preattentive processing of the color of the whole house (Wolfe et al., 1994).

Mean error rates for each condition are presented in the Appendix. Error rates in the part-feature and wholefeature conditions and in the part-whole blank trials were quite low, but error rates in the part-whole target trials were quite high. The mean errors for those trials rose from $12.4 \%$ in set size 4 to $30.4 \%$ in set size 16 . For this condition, it seems that $3,000 \mathrm{msec}$ were simply not always enough time to find the target, especially when the set size became large. If the subjects had been given more time in which to respond, the RTs would almost certainly have been higher and the corresponding slope steeper.

Since both the vertical rectangle and the tilted bar of the part-whole conjunction target can be found without recourse to a serial, self-terminating search of all items, the inefficiency of orientation $\times$ orientation search is most likely due to an inability to appropriately combine the information about the two feature attributes. Nevertheless, since the slopes in the whole-feature condition

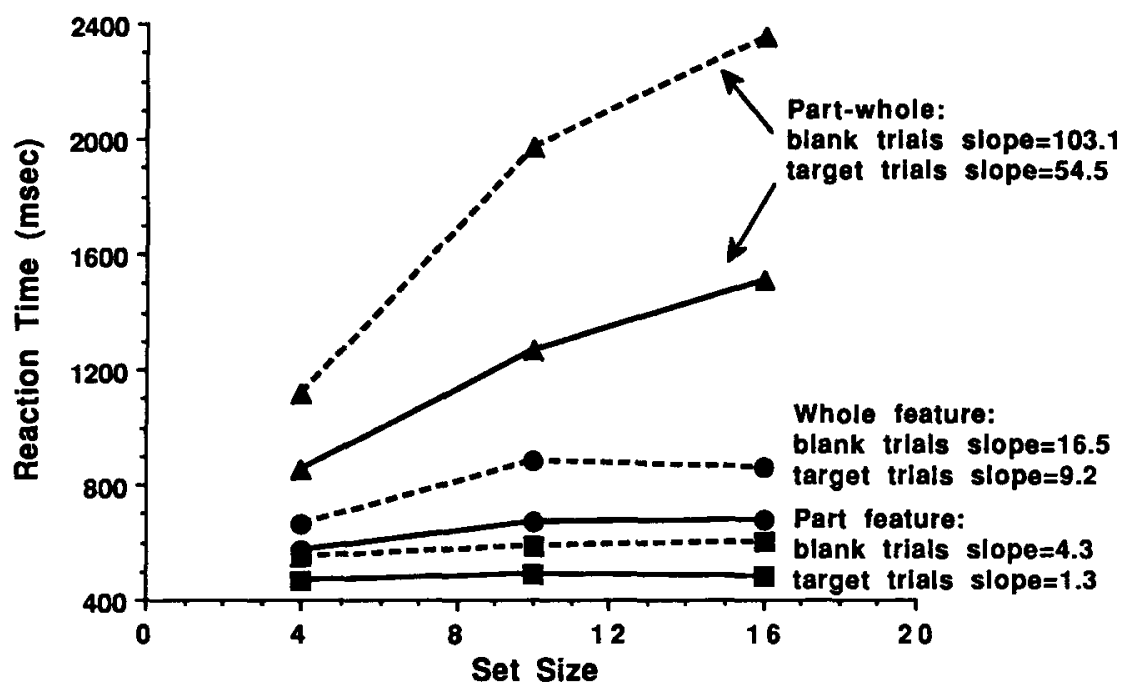

Figure 8. Results for Experiment 4, showing very inefficient search for orientation $\times$ orientation conjunctions, even in a part-whole configuration. 
are rather steep, it is hard to eliminate inefficient feature search as the basis for inefficient conjunction search. In an effort to strengthen the case, the basic experiment was repeated with new stimuli.

\section{EXPERIMENT 5 "Skinny Letters"}

Combinations of orientations make new forms in ways that combinations of such features as color do not. In Experiment 5, we attempted to exploit this source of information in an effort to produce an efficient partwhole orientation $\times$ orientation search.

\section{Method}

Figure 9 shows the stimuli used in Experiment 5. The stimuli look like letters of a sort. Each stimulus item was constructed from two long parallel bars, both horizontal or both vertical, connected in the center by a short horizontal or $-45^{\circ}$ tilted bar. The two long parallel bars were close enough to each other to create the impression of an oriented whole. In the part-whole condition, subjects searched for the item with long, vertical bars connected by a short, tilted bar among items with long, vertical bars connected by a short, horizontal bar and items with long, horizontal bars connected by a short, tilted bar. In the whole-feature search, subjects searched for just the long, vertical bars. In the part-feature search, subjects searched for just the short, tilted bar. The stimulus array subtended an area $15.3^{\circ} \times 15.3^{\circ}$ at the $62-\mathrm{cm}$ viewing distance. The maximum presentation time was $4,500 \mathrm{msec}$. The smallest set size used in this experiment was 6 rather than 4 as in all the previous experiments. The methods were otherwise similar to those in Experiment 1

Ten new subjecs were tested.

\section{Results and Discussion}

Average RT $\times$ set-size functions are shown in Figure 10. Again, the part-whole search was very inefficient, with slopes comparable to standard serial-search slopes. In contrast with Experiment 4, both the part- and
Part-Whole Condition

Target - Vertical item with tilted bar

Target Distractors
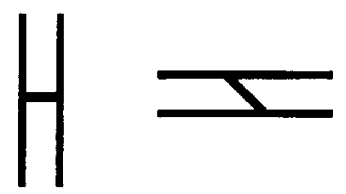

Whole Feature Condition

Target - Vertical item

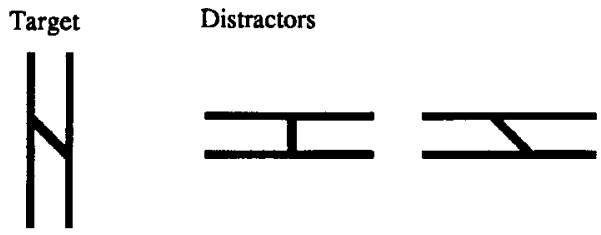

Part Feature Condition

Target - Tilted bar

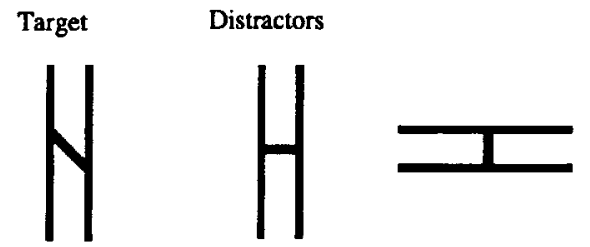

Figure 9. Stimuli for Experiment 5: Orientation $\times$ orientation conjunctions where parts and wholes are more clearly bound together into an item (a "skinny letter").

whole-feature conditions in the present experiment had very shallow slopes for target trials (target-trial slopes of 3.2 and $1.2 \mathrm{msec} /$ item, respectively). This demonstrates that the orientation differences here were large enough

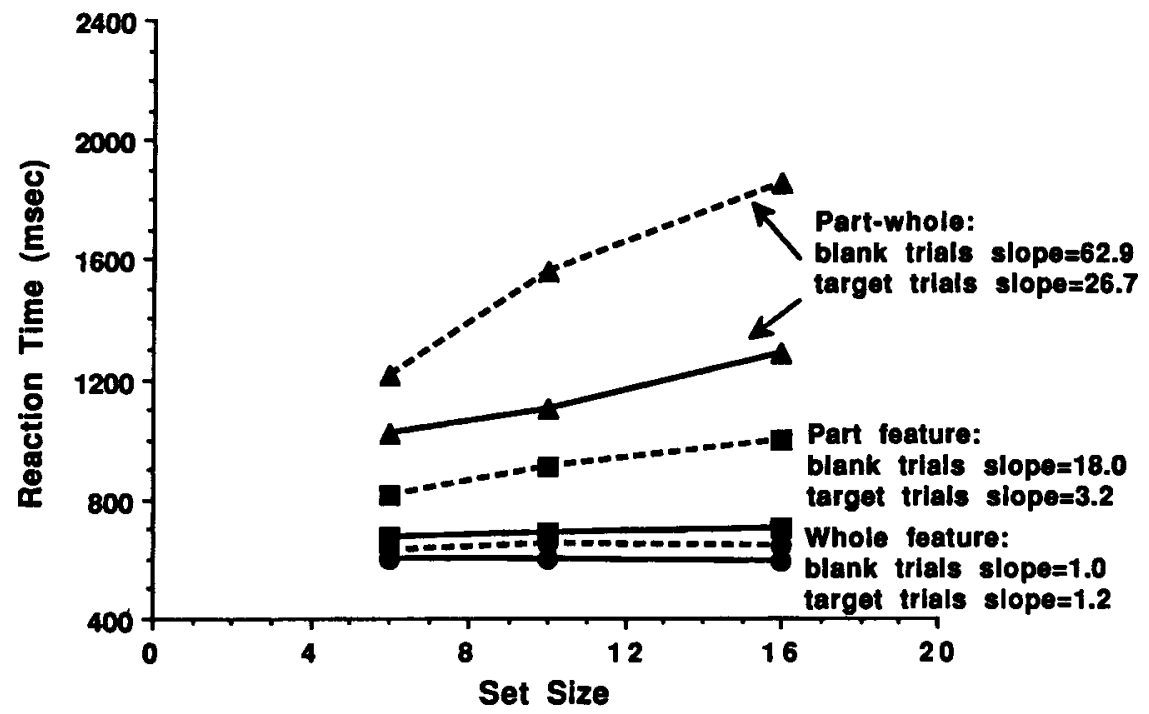

Figure 10. Results for Experiment 5, replicating very inefficient search for orientation $\times$ orientation conjunctions, even in a part-whole configuration. 


\section{Part-Whole Condition}

Target - Vertical item with tilted bars

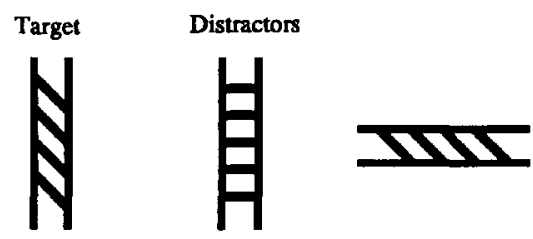

Whole Feature Condition

Target - Vertical item

Target Distractors

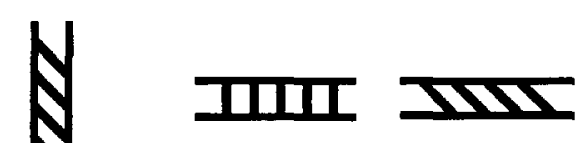

Part Feature Condition

Target - Tilted bars

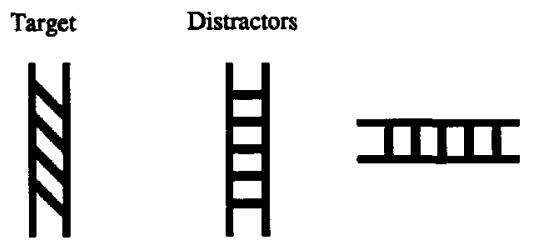

Figure 11. Stimuli for Experiment 6: Another effort to create stimuli for efficient part-whole orientation $\times$ orientation conjunction search.

to permit efficient search. In this case, the failure to efficiently search for an orientation $\times$ orientation conjunction could not be attributed to an inability to search for one or both of the component orientations. Moreover, in Experiment 4, the rectangle and the enclosed bar could be seen as two items rather than as a whole item with a part. In Experiment 5, each "skinny letter" is clearly one perceptual item.

Mean error rates for each condition are presented in the Appendix. Subjects made few errors in this experiment.

\section{EXPERIMENT 6 Ladders}

Ever mindful of the problems in proving the negative hypothesis that part-whole orientation $X$ orientation searches are never efficient, in Experiment 6 we attempted once again to create an efficient hierarchical orientation $\times$ orientation conjunction search. We tried to improve on the stimuli in Experiment 5 by making the orientation of the "parts" in those stimuli more salient. To this end, we connected the two long, parallel bars in each item with several short bars instead of just one. We thought that the addition of several tilted bars might increase the saliency of that feature. In addition, the parallel bars were moved slightly closer to each other to make each item as a whole slightly more oriented.

\section{Method}

The modified stimuli are shown in Figure 11. The target was always a vertical "ladder" with tilted "rungs." In the part-whole condition, the target was defined by the conjunction of ladder and rung orientation. In the feature conditions, the target was defined by either the ladder or the rung orientation in isolation. The stimulus array subtended an area of $17^{\circ} \times 17^{\circ}$ at the $57-\mathrm{cm}$ viewing distance. Set sizes were 4,10 , and 16. Search conditions and methods were otherwise identical to those in Experiment 5.

Ten new subjects were tested.

\section{Results and Discussion}

Figure 12 illustrates average RT $\times$ set size functions for each condition for all 10 subjects. Part-whole slopes were again typical of serial-search slopes. Feature-search slopes were again shallow. Mean error rates for each

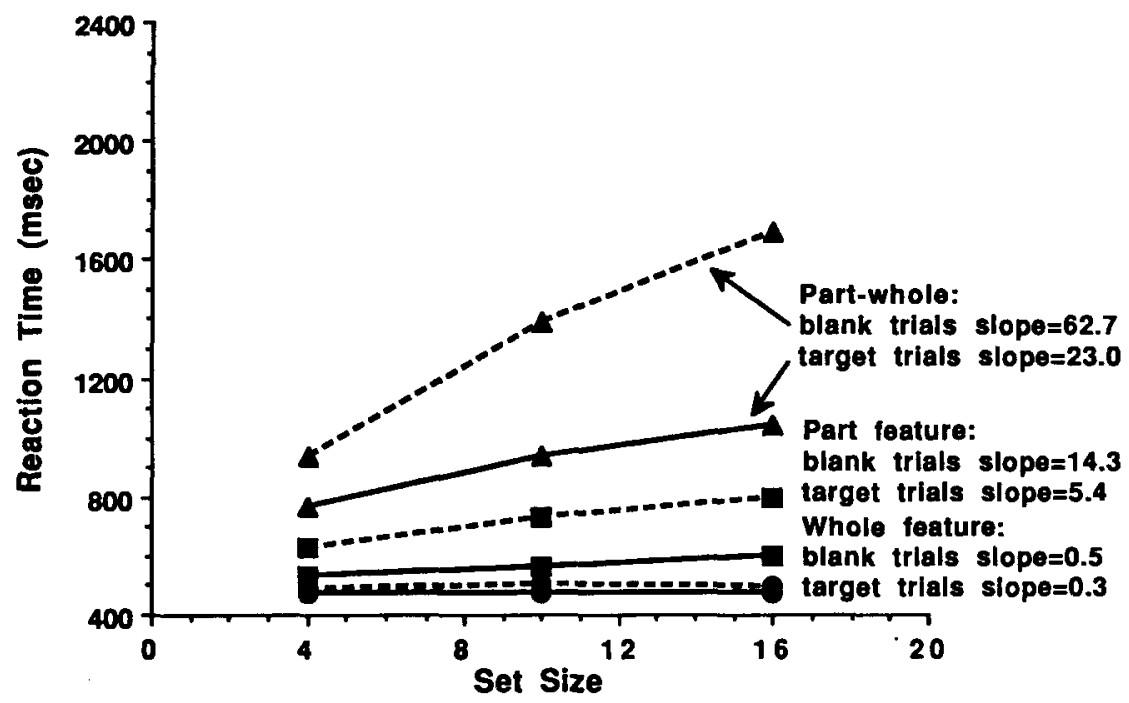

Figure 12. Results for Experiment 6, showing another failure to produce efficient search for orientation $\times$ orientation conjunctions. 
condition are presented in the Appendix. Subjects made few errors in this experiment.

\section{GENERAL DISCUSSION}

In three separate experiments, we were unable to produce an efficient hierarchical orientation $\times$ orientation conjunction search. While we must, of course, be cautious about drawing conclusions from negative findings, it seems that information about hierarchical relationships between regions of items cannot be used to make search efficient in orientation $\times$ orientation conjunction search tasks. We have designed many other stimuli for hierarchical orientation $\times$ orientation search tasks in an effort to disprove this negative hypothesis. Some of these are shown in Figure 13.

In all cases, pilot data indicate that the orientation $\times$ orientation search remains difficult even for very experienced subjects. In many cases, one of the two underlying feature searches is hard, perhaps because it is difficult to have two different, preattentively detectable orientations in approximately the same location. The subjective impression is that the more salient orientation interferes with search for the less salient orientation. This problem is not encountered when designing stimuli for a hierarchical color $\times$ color task. As noted above, a red house does not interfere with the ability to identify the color of a yellow window in the way that a tilted whole seems to interfere with the ability to identify the orientation of a part. It is possible that the inefficiency of these orientation feature searches and the inefficiency of the orientation $X$ orientation conjunction searches have the same underlying cause. If orientation is not represented hierarchically at a preattentive stage, irrelevant orientations from one scale can interfere with search for a particular orientation at another scale. While this seems a plausible account, at present we do not have enough evidence to present this as anything but a hypothesis.

The six experiments reported here extend our knowledge about the role of hierarchical relationships between regions of an item in visual search tasks. Moreover, they demonstrate that, when it comes to visual search, all features are not created equal. Subjects can use information about hierarchical relationships between regions in stimulus items to help them search efficiently for targets defined by conjunctions of two sizes or two colors. Searches for conjunctions of these same features are inefficient when the regions are not arranged hierarchically. For orientation, hierarchical relationships appear to be useless and irrelevant. We invite others to design successful, efficient orientation $\times$ orientation searches. However, on the basis of our experience, we will be surprised if anyone succeeds.

According to the guided search model (Wolfe, 1994), search will be efficient when attention can be guided to the target by information from one or more parallelfeature processors. If the search target is defined by the conjunction of two instances of the same type of feature, the feature processor in question will provide useful information only if it directs attention more vigorously to the location with both target attributes than to locations with only one target attribute. To do this, the singlefeature processor must sum the activations for the two attributes. The puzzle presented by these data is to explain why attention can be guided to some within-feature conjunctions and not to others. For color and for size, information about two target colors or two target sizes can be used to guide attention when the target attributes characterize regions of a stimulus that are in a hierarchical relationship to one another. Wolfe et al. (1994) have suggested that hierarchical information may lead to the summing of the activations of the two target attributes by causing the creation of temporary, separate representations for attributes at each level of the hierarchy (cf. object files: Treisman, Kahneman, \& Burkell, 1983; Kahneman, Treisman, \& Burkell, 1983). Without hierarchical

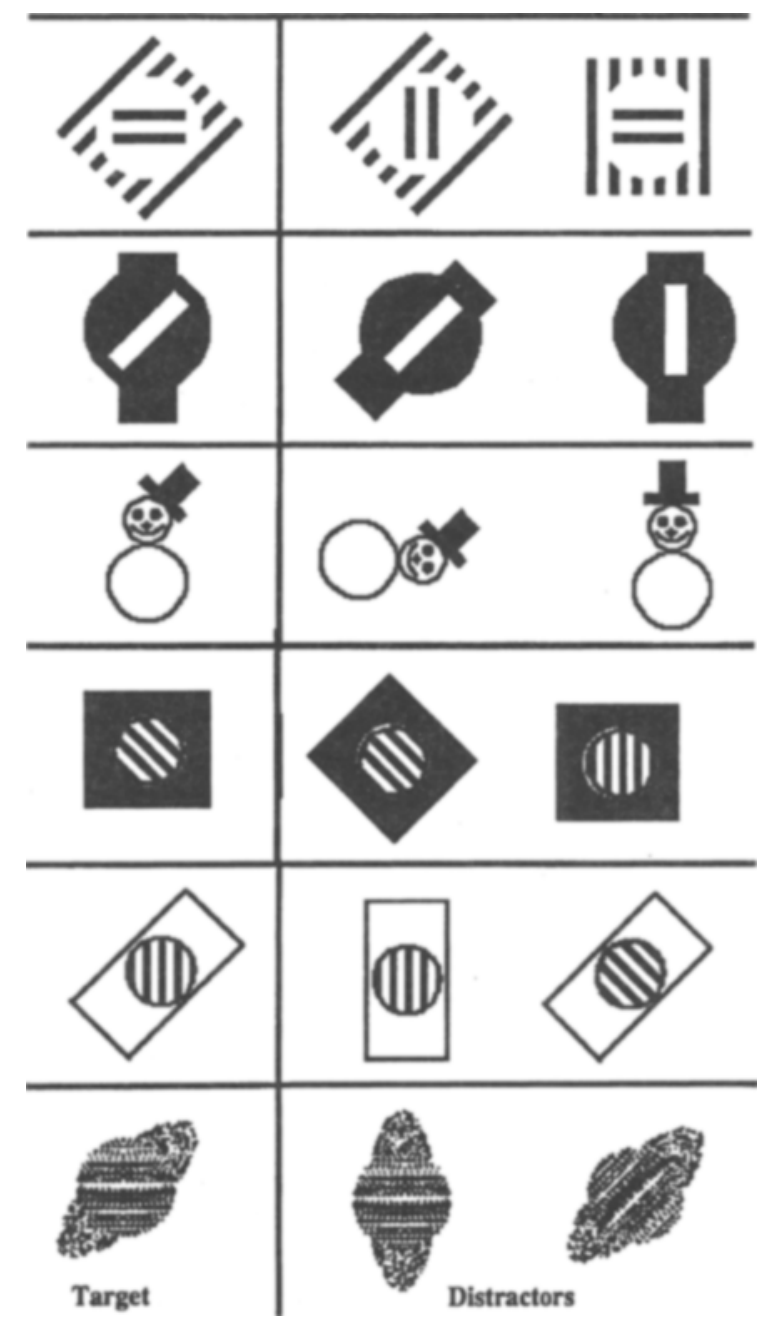

Figure 13. A variety of other stimuli that failed to produce efficient search in pilot experiments. 
organization, there are no separate representations, activations are not summed, and search is inefficient.

The orientation processor, however, seems unable to exploit hierarchical information. The present experiments do not address the reason for this distinction between color and size, on the one hand, and orientation, on the other. Nevertheless, we can speculate. Colors and sizes are relatively invariant properties of objects. Orientations of objects, on the other hand, are often easily altered. Consider a search for a book. A yellow book with black print on the spine remains a yellow book with black print under most circumstances. A large book with small print remains a large book with small print under most circumstances. However, there is no guarantee that a book that at one time was tilted $45^{\circ}$ from vertical with print tilted $45^{\circ}$ in the opposite direction will still have the same orientations at present. It may be that the visual system declined to invest in the parallel processing of hierarchical orientation information because other, more invariant cues, related to orientation, are usually available. Wolfe and Friedman-Hill (1992) have shown that angles formed by oriented lines can be detected in parallel in visual search. The stimuli used in the present experiments were designed so that the angle formed by the orientations of the part and of the whole would not distinguish the target from the distractors. It is this angle, however, and not absolute orientation that would be resistant to change in natural targets. To return to the example above, without radical changes in the object, the print on the book will always be at a $90^{\circ}$ angle to the book as a whole. The use of angle information may make the use of hierarchical orientation information unnecessary in natural search situations.

There is at least one other situation in which orientation differences are lost, while color differences, at least, are preserved. Nothdurft $(1991,1993)$ has done a number of experiments in which subjects see three or four odd items in a field of identical items. For example, all of the background items might be green vertical lines. If three items are oriented horizontally, the resulting triangle is easily seen (Nothdurft, 1991). Similarly, if three items are red, the triangle is easily seen. Suppose, however, that each vertex of the triangle is formed by a different orientation or a different color. A triangle formed with one horizontal, one left-oblique, and one right-oblique vertex is easily seen. A triangle formed by one red, one blue, and one yellow vertex can be traced out, of course, but it does not emerge as effortlessly as the orientation version (Nothdurft, 1993; Wolfe, Chun, \& Friedman-Hill, 1995). Apparently, variously oriented singletons can group together more readily than variously colored singletons.

In summary, preattentive processing of color and size can be influenced by the configuration of the items being processed. Subjects can search efficiently for conjunctions of two colors or two sizes if one color or size labels the whole item while the other labels a part. By contrast, all searches for conjunctions of two orientations are inefficient, suggesting that parallel processing of orientation is qualitatively different from processing for color or form.

\section{REFERENCES}

DUNCAN, J. (1993). Coordination of what and where systems in the visual control of behaviour. Perception, 22, 1261-1270.

EnNs, J. T., Ochs, E. P., \& Rensink, R. A. (1990). Vsearch: Macintosh software for experiments in visual search. Behavior Research Methods, Instruments, \& Computers, 22, 118-122.

Kahneman, D., Treisman, A., \& Burkell, J. (1983). The cost of visual filtering. Journal of Experimental Psychology: Human Perception \& Performance, 4, 510-522.

KIMCHI, R. (1992). Primacy of wholistic processing and global/local paradigm: A critical review. Psychological Bulletin, 112, 24-38.

KWAK, H., Dagenbach, D., \& EgETH, H. (1991). Further evidence for a time-independent shift of the focus of attention. Perception \& Psychophysics, 49, 473-480.

NoTHDURFT, H. C. (1991). Texture segmentation and pop-out from orientation contrast. Vision Research, 31, 1073-1078.

NothduRFT, H. C. (1993). The role of features in preattentive vision: Comparison of orientation, motion and color cues. Vision Research, 33, 1937-1958.

O'NeILl, P., \& WolfE, J. M. (1994). Mechanisms of visual search revealed by individual differences. Investigative Ophthalmology \& Visual Science, 35, 1328.

TownSEND, J. T. (1990). Serial and parallel processing: Sometimes they look like Tweedledum and Tweedledee but they can (and should) be distinguished. Psychological Science, 1, 46-54.

Treisman, A., \& Gelade, G. (1980). A feature-integration theory of attention. Cognitive Psychology, 12, 97-136.

TREISMAN, A., \& Gormican, S. (1988). Feature analysis in early vision: Evidence from search asymmetries. Psychological Review, 95, 15-48.

Treisman, A., Kahneman, D., \& Burkell, J. (1983). Perceptual objects and the cost of filtering. Perception \& Psychophysics, 33, 527-532.

WoLFE, J. M. (1994). Guided search 2.0: A revised model of visual search. Psychonomic Bulletin \& Review, 1, 202-238.

Wolfe, J. M., CAVE, K. R., \& Franzel, S. L. (1989). Guided search: An alternative to the feature integration model for visual search. Journal of Experimental Psychology: Human Perception \& Performance, 15, 419-433.

Wolfe, J. M., Chun, M. M., \& Friedman-Hill, S. R. (1995). Making use of texton gradients: Visual search and perceptual grouping exploit the same parallel processes in different ways. In T. V. Papathomas, C. Chubb, A. Gorea, \& E. Kowler (Eds.), Early vision and beyond (pp. 189-198). Cambridge, MA: MIT Press.

Wolfe, J. M., \& FriedmaN-Hill, S. R. (1992). Visual search for orientation: The role of angular relations between targets and distractors. Spatial Vision, 6, 199-208.

Wol.fe, J. M., Friedman-Hill, S. R., \& Bilsky, A. B. (1994). Parallel processing of part/whole information in visual search tasks. Perception \& Psychophysics, 55, 537-550.

Wolfe, J. M., Yu, K. P., Stewart, M. I., Shorter, A. D., FriedmanHILL, S. R., \& CAVE, K. R. (1990). Limitations on the parallel guidance of visual search: Color $X$ color and orientation $X$ orientation conjunctions. Journal of Experimental Psychology: Human Perception \& Performance, 16, 879-892.

\section{NOTES}

1. For the sake of convenience, we refer to the subordinate and superordinate regions of these hierarchical stimuli as the "part" and the "whole," respectively. In this context, the terms "part" and "whole" should be understood only in a very restricted sense. We are not claiming that the parallel ("preattentive") analysis of items gives a full, perceptual description of the parts and wholes of objects.

2 . Townsend has pointed out that RT $\times$ set-size functions are not, by themselves, enough to determine if a process is serial or parallel. Limited-capacity parallel models can make the same predictions as serial models. Instead of assuming that each item is processed in series, limited-capacity parallel models assume that all items are processed at once but that some processing resource must be spread over all items. This makes larger set sizes slower. With appropriate choices of parameters, these models predict the results as well as any serial model. 
APPENDIX

Mean Error Rates (Pencent) for Each Experiment Condition $\times$ Trial Type $\times$ Set Size

\begin{tabular}{|c|c|c|c|c|c|c|c|c|c|}
\hline \multirow[b]{2}{*}{ Experiment } & \multirow[b]{2}{*}{ Condition } & \multicolumn{4}{|c|}{ Target Set Sizes } & \multicolumn{4}{|c|}{ Blank Set Sizes } \\
\hline & & 4 & 6 & 10 & 16 & 4 & 6 & 10 & 16 \\
\hline \multirow[t]{3}{*}{1} & Feature & 1.4 & & 3.0 & 3.2 & 2.2 & & 1.0 & 1.2 \\
\hline & Part-whole & 2.2 & & 2.2 & 5.0 & 2.4 & & 2.2 & 1.2 \\
\hline & Part-part & 7.4 & & 8.4 & 14.4 & 0.6 & & 0.8 & 1.6 \\
\hline \multirow[t]{3}{*}{2} & Feature & 2.0 & & 2.2 & 2.4 & 3.2 & & 1.2 & 1.4 \\
\hline & Part-whole & 0.8 & & 2.2 & 3.4 & 1.8 & & 0.6 & 0.8 \\
\hline & Part-part & 6.6 & & 9.6 & 15.0 & 0.8 & & 0.4 & 2.8 \\
\hline \multirow[t]{3}{*}{3} & Feature & 1.8 & & 2.4 & 3.2 & 2.6 & & 1.4 & 1.0 \\
\hline & Part-whole & 2.8 & & 6.0 & 6.6 & 2.0 & & 1.2 & 1.8 \\
\hline & Part-part & 5.8 & & 5.0 & 9.8 & 1.8 & & 0.4 & 1.2 \\
\hline \multirow[t]{3}{*}{4} & Part feature & 1.0 & & 1.0 & 2.2 & 2.0 & & 0.6 & 1.0 \\
\hline & Whole feature & 2.2 & & 5.0 & 7.6 & 2.4 & & 1.8 & 0.8 \\
\hline & Part-whole & 12.4 & & 21.4 & 30.4 & 1.0 & & 1.4 & 6.6 \\
\hline \multirow[t]{3}{*}{5} & Part feature & & 2.0 & 1.8 & 2.4 & & 1.2 & 0.2 & 0.8 \\
\hline & Whole feature & & 0.8 & 1.2 & 2.8 & & 0.6 & 0.4 & 0.8 \\
\hline & Part-whole & & 3.4 & 4.6 & 6.4 & & 2.2 & 1.2 & 1.6 \\
\hline \multirow[t]{3}{*}{6} & Part feature & 1.2 & & 1.2 & 2.2 & 0.6 & & 0.0 & 0.2 \\
\hline & Whole feature & 1.6 & & 0.8 & 0.6 & 0.2 & & 0.6 & 1.0 \\
\hline & Part-whole & 3.4 & & 4.4 & 6.2 & 1.6 & & 0.4 & 0.4 \\
\hline
\end{tabular}

(Manuscript received August 12, 1994;

revision accepted for publication January 4, 1995.) 